\title{
Lernen im Aktivismus: Ein Erfahrungsbericht
}

Derzeit studiere ich im Bachelorstudiengang Global Environmental and Sustainability Studies mit dem Nebenfach Politikwissenschaften an der Leuphana Universität Lüneburg. Seit Beginn meines Studiums vor drei Jahren bin ich bei unterschiedlichen Initiativen und politischen Jugendgruppen ehrenamtlich aktiv. Im Zuge dessen habe ich als Teil eines Teams die Vernetzungsinitiative Lebendiges Lüneburg mit aufgebaut, die das Ziel verfolgt, das zivilgesellschaftliche Engagement in Lüneburg auf verschiedenste Art und Weise zu stärken und zu vernetzen.

Motivation - mein Anfang: „Der Klimawandel wartet nicht, bis dein Bachelor fertig ist.", steht auf vielen Aufklebern am Campusgelände. Der Wunsch, einen Beitrag zur Lösung dieser und anderer Herausforderungen zu leisten, bekam für mich in der Universität eine neue Dringlichkeit, da ich mich sowohl durch mein Studienfach als auch durch den Austausch mit meinen engagierten Kommiliton/-innen, mehr mit den drastischen Auswirkungen des Klimawandels befasste. Mir reichte es nicht, in Vorlesungen und Seminaren Wissen zu erwerben und nach und nach Fähigkeiten zu entwickeln, mit denen ich mich im Anschluss an das Studium in die Gesellschaft einbringen kann. Es entstand der Wunsch, direkt tätig zu werden. Im Umfeld meiner Universität gibt es ein überwältigendes Angebot von Initiativen und Gruppen. Einen Überblick darüber zu erlangen und herauszufinden, welche Initiative für mich die richtige wäre, schien mir kaum möglich. So kam es, dass persönliche Kontakte den Ausschlag gaben. Freund/-innen, die bereits engagiert waren, kamen auf mich zu: „Komm doch mal mit, das ist doch voll dein Ding!“, oder „Wir suchen noch Menschen, wäre das nicht was für dich?" . Recht hatten sie und damit auch einen entscheidenden Einfluss auf meine weitere persönliche Entwicklung. Die wichtigsten Faktoren für mein aktivistisches Engagement waren und sind für mich daher vor allem meine Mitmenschen und der Wunsch, gemeinsam mit ihnen zu einer sozial und ökologisch gerechten Welt beizutragen. Der Rahmen aktivistischer Tätigkeit, über den ich in diesem Artikel schreibe, ist sehr weit gefasst und beinhaltet für mich nicht nur Demonstrationen oder die direkte Aktion, sondern auch alle weiteren, (un-)organisierten Tätigkeiten für einen gesellschaftlichen und politischen Wandel. Meine persönliche Erfahrung bezieht sich dabei vor allem auf mein Aktivsein in einer Initiative für die Vernetzung und Stärkung des lokalen, zivilgesellschaftlichen Engagements, einigen politischen Gruppen und meine Teilnahme bei Demonstrationen und Aktionen zivilen Ungehorsams der Klimagerechtigkeitsbewegung. Der dritte motivierende Faktor neben meinen Mitmenschen und dem Wunsch nach nachhaltigem Wandel, war für mich die Absicht, in der aktivistischen Tätigkeit Dinge zu erlernen, die ich an der Universität vermisste. Auch im Lüneburger Mitwirk-O-Mat (einer Web-Anwendung, die wir im Rahmen der Vernetzungsinitiative Lebendiges Lüneburg umsetzten, um Menschen den Einstieg in ein Engagement zu vereinfachen) geben $71 \%$ der Nutzer/-innen an, sich durch Engagement weiterbilden zu wollen. Die Art und Weise, wie das Lernen im Aktivismus funktioniert und welche Inhalte dabei relevant wurden, steht im Fokus dieses Beitrags.

Anwendungsorientierung: Die wichtigste Eigenschaft des Lernens im Aktivismus ist für mich, dass dieser mich dazu bringt, Gelerntes stets wiederzugeben und direkt anzuwenden. Es geht also weniger um den Input, den ich aufnehme, sondern mehr um den Output, den ich wiedergebe. Zum Beispiel sammle ich an einem Stand in der Fußgängerzone Unterschriften und komme mit immer wieder unterschiedlichen Menschen ins Gespräch. Solche Gespräche und Situationen wiederholen sich niemals in gleicher, aber oft in ähnlicher Form zu verschiedenen Aspekten eines großen Themengebiets. Viele solcher kleinen „Prüfungen“, so ist mein Eindruck, führen dazu, dass ich die dabei behandelten Informationen besser im Gedächtnis behalten kann als das, was ich für einmalige Prüfungen gelernt habe. Auch bei praktischen Fähigkeiten ist es so, dass diese in den meisten Fällen direkt erprobt werden und zur Anwendung kommen.

Lernen, um zu wissen und Lernen, um zu können: Die Auseinandersetzung mit theoretischen Inhalten ist im Aktivismus unausweichlich. Welche Inhalte das sind, hängt natürlich von dem Anliegen ab, für das ein Mensch aktiv ist. Bei mir waren und sind es vor allem die Themen Klimagerechtigkeit und Bürger/-innenbeteiligung, mit denen ich mich befasse und in denen sich für mich im Laufe meines Engagements auch Veränderungen ergaben. Seitdem ich mich engagiere, suche und 
finde ich zudem auch in meinen Kursen an der Universität Möglichkeiten, mich mit für mein Engagement relevanten Theorien und Inhalten zu befassen. Von daher haben sich inhaltliche Fortschritte für mich aus der Verzahnung von Aktivismus, universitärer Lehre oder freizeitlichem Vertiefen ergeben. Viele, vor allem praktische Fähigkeiten sind jedoch klar auf die Anforderungen in meinem Engagement zurückzuführen, z.B. das Organisieren von Veranstaltungen, das Moderieren von Plenen, das Schreiben von Förderanträgen, das Erstellen von Websites, das Durchfließen von Polizeiketten sowie das Lösen zwischenmenschlicher Konflikte. Die Vielfalt der im Aktivismus geforderten und gebildeten Fähigkeiten variiert je nach Engagement, umfasst jedoch in den meisten Fällen eine Bandbreite von sowohl technischen als auch zwischenmenschlichen Fähigkeiten. Da es im Aktivismus gerade darum geht, etwas zu tun, zu bewegen und zu verändern, ist es wohl wenig überraschend, dass dieser Teil derjenige ist, der mir im Rückblick als der wichtigere und lehrreichere erscheint. Während ich einen Teil, der die theoretischen Konzepte, Fakten und Ideen umfasst, als Lernen, um etwas zu wissen charakterisieren würde, sehe ich den anderen Teil als ein Lernen, um etwas zu können. Durch die praktischen Erfahrungen konnte ich direkt erproben, was ich meinte theoretisch tun zu können.

Voneinander lernen: Bei den oben genannten Beispielen wird bereits deutlich, dass das Lernen im Aktivismus vor allem ein voneinander Lernen ist. Dabei kann ich drei Personengruppen unterscheiden: Erstens, das Lernen von den eigenen Mitstreitenden, zweitens, von politischen Gegner/-innen und drittens, von Fremden. Zuletzt gehört auch noch das Vernetzen zu den Dingen, die ich im Aktivismus lerne. Dadurch, dass ich als Teil der Gruppen, in denen ich aktiv bin, in ein aktivistisches Umfeld eingebunden werde, lerne ich wichtige Akteur/-innen und Institutionen dieses Bereichs kennen und erhalte Einblicke in Strukturen, die mir ansonsten verborgen geblieben wären. Dieses Wissen kann mir helfen, mich in Zukunft mit meinen Ideen und Anliegen an die richtigen Stellen zu wenden oder als Vermittler für andere Menschen und Initiativen zu fungieren. Möglichkeiten, von den eigenen Mitstreiter/-innen zu lernen, ergeben sich im Aktivismus häufig, da es der gesamten Bewegung hilft, wenn Menschen bereit sind, ihr Wissen an ihre Mitstreiter/-innen weiterzugeben. Vor allem auf Versammlungen wie Klimacamps gibt es oft Workshops oder ähnliche Formate, bei denen Wissen und Fähigkeiten vermittelt werden. Aber auch im aktivistischen Alltag, in der Initiative oder dem persönlichen Kontakt ergeben sich ständig Situationen, in denen ich von meinen Mitstreitenden lerne und sie mir zeigen, wie sie zum Beispiel eine Website designen oder Menschen in der Fußgängerzone ansprechen. Außerdem stehe ich mit meinen Mitstreitenden im ständigen Austausch über Inhalte und tagesaktuelle Themen. Einige der für mich wichtigsten Impulse zu Theorien, Büchern und Perspektiven habe ich von meinen Freund/-innen im Aktivismus erhalten. Somit macht das voneinander Lernen unter Mitstreiter/-innen den größten Teil des Lernens im Aktivismus aus. Dann wiederum bringt mich der Aktivismus auch in Kontakt mit Menschen, die meinem Standpunkt widersprechen und politische Gegner/-innen sind. Mir hat der Austausch mit politischen Gegner/-innen stets geholfen, meine eigene Position zu überprüfen und zu schärfen, aber auch zu verstehen, weshalb andere dagegen sind. Manchen Menschen ist es aller- dings gar nicht möglich, mit politischen Gegner/-innen auf Augenhöhe zu sprechen, da sie von ihnen aufgrund verschiedenster Merkmale diskriminiert werden. Wenn die Möglichkeit jedoch gegeben ist, halte ich es für wichtig, diese Gespräche zu suchen, von diesem Austausch zu lernen und dabei auf einen Abbau diskriminierender Verhaltensweisen hinzuwirken. Zuletzt ergibt es sich im Aktivismus auch, vom Wissen und von den Erfahrungen fremder Menschen zu lernen. In Lüneburg nutzen wir dafür beispielsweise die Plattform Lünepedia, über die Menschen ihr Wissen über lokal relevante Orte, Strukturen und Möglichkeiten verfügbar machen können.

Bei mir ist es so, dass der Aktivismus und mein freiwilliges Engagement mit der Zeit wichtiger wurden als das Lernen an der Universität. Schließlich geht es auch um wichtige Herausforderungen, zu deren Lösung ich beitragen möchte: Das Verhindern der Klimakatastrophe und die gesellschaftliche Teilhabe aller Menschen zu ermöglichen. Angesichts dessen, dass diese Herausforderungen so groß sind und gleichzeitig die Zahl der vor Ort aktiven Menschen oft überschaubar bleibt, steigt die Gefahr, sich selbst immer mehr Aufgaben aufzubürden, und sich so zu überfordern, zu überarbeiten und auszubrennen. Um langfristig und nachhaltig aktivistisch tätig bleiben zu können, ist ein achtsamer Umgang mit sich selbst, mit anderen Menschen sowie den bevorstehenden Herausforderungen nötig. Genauso, wie Aktivismus eine zusätzliche Bereicherung zum Lernen in der Universität sein kann, kann Aktivismus auch eine zusätzliche physische oder psychische Belastung werden. Achtsamkeit und damit einhergehende Techniken zur Bewältigung dieser Belastungen werden daher bereits an vielen Orten im Aktivismus vermittelt und praktiziert und sind wichtig, um zu einer lebendigen Bewegung beizutragen.

Alles in allem bringt mich der Aktivismus von einem Zustand des „Ich könnte das!“, in dem alles Wissen theoretisch vorhanden ist, zu einem selbstbewussten „Ich kann das!“, in dem das Wissen praktisch erprobt und fest in mir selbst verankert ist. Welchen Einfluss mein eigenes Handeln dabei auf die Bewegung und die gesetzten Ziele hat, bleibt allerdings sehr diffus. Dies gilt insbesondere im Hinblick auf globale Phänomene wie die Klimagerechtigkeitsbewegung. Vor Ort und auch darüber hinaus konnte ich im Team von Lebendiges Lüneburg zwar einige Ideen, beispielsweise den Mitwirk-O-Mat, umsetzen und dabei auch eine Selbstwirksamkeit erfahren. Doch auch hier ist das Ehrenamt noch lange nicht so sichtbar und vernetzt, wie wir es uns wünschen würden. Das, worauf ich eindeutiger zurückschauen kann, ist, dass mir persönlich das aktivistische Engagement viel gebracht hat und ich an den Herausforderungen, die mein Engagement für mich bereithielt, gewachsen bin.

Jonas Schröder doi.org/10.31244/zep.2021.03.06 